\title{
A b-continuidade de grafos com cintura alta.
}

\author{
Allen Ibiapina , Ana Silva \\ ${ }^{1}$ Departamento de Matemática - Universidade Federal do Ceará (UFC) \\ Fortaleza - CE - Brazil \\ ParGO - Paralelismo, Grafos e Otimização \\ allenr.roossim@gmail.com, anasilva@mat.ufc.br
}

\begin{abstract}
A b-coloring of a graph is a proper coloring such that each color class has at least one vertex which is adjacent to each other color class. The $b$-spectrum of $G$ is the set $S_{b}(G)$ of integers $k$ such $G$ has a b-coloring with $k$ colors and $b(G)=\max S_{b}(G)$ is the b-chromatic number of $G$. A graph is $b$-continous if $S_{b}(G)=[\chi(G), b(G)] \cap \mathbb{Z}$. An infinite number of graphs that are not b-continuous is known. Also it is known that graphs with girth at least 10 are b-continuous. In this article, we prove that graphs with girth at least 8 are $b$-continuous and that the b-spectrum of graphs with girth at least 7 contains the integers between $2 \chi(G)$ and $b(G)$.
\end{abstract}

Resumo. Uma b-coloração de um grafo é uma coloração própria, tal que cada classe de cor possui um vértice que é vizinho de pelo menos um vértice das outras classes de cores. O b-espectro de $G$ é o conjunto $S_{b}(G)$ dos inteiros $k$ tais que $G$ tem uma b-coloraçao com $k$ cores e $b(G)=\max S_{b}(G)$ é o número b-cromático de $G$. Um grafo é b-contínuo se $S_{b}(G)=[\chi(G), b(G)] \cap \mathbb{Z} . \quad E$ conhecida uma infinidade de grafos que não são b-contínuos. Também é sabido que grafos com cintura maior ou igual a 10 são b-contínuos. Neste artigo, mostramos que grafos com cintura pelo menos 8 são b-contínuos e que o $b$ espectro de grafos com cintura pelo menos 7 contém os inteiros entre $2 \chi(G) e$ $b(G)$.

\section{Introdução}

Seja $G$ um grafo simples ${ }^{1}$. Uma $k$-coloração própria de $\mathrm{G}$, doravante chamada apenas de $k$-coloração, é uma função $\psi: V(G) \rightarrow \mathbb{N}$ tal que $|\psi(V(G))|=k$ e $\psi(u) \neq \psi(v)$ sempre que $u v \in E(G)$. Dizemos que $u \in V(G)$ é um b-vértice em $\psi($ de cor $\psi(u)$ ) se para toda cor $c \in \psi(V(G)) \backslash\{\psi(u)\}$, existe $v$ vizinho de $u$ tal que $\psi(v)=c$. Se para uma classe de cor $c \in \psi(V(G))$, não existem b-vértices da cor $c$, podemos obter uma $(k-1)$-coloração recolorindo cada vértice da cor $c$ com uma cor de $\psi(V(G)) \backslash\{c\}$ que não aparece em sua vizinhança; dizemos que essa nova coloração é obtida de $\psi$ pela limpeza da cor $c$. Para uma coloração que não podemos aplicar esse algorítmo para diminuir a quantidade de cores, toda classe de cor $c$ possui um b-vértice. Uma coloração assim é chamada de $b$ coloração. Como o problema de coloração é NP-completo, nem sempre uma b-coloração usa apenas $\chi(G)$ cores. Note que toda coloração de $G$ usando $\chi(G)$ cores é uma bcoloração, caso contrário poderíamos diminuir o número de cores usadas. Por isso, a

\footnotetext{
*Este trabalho foi parcialmente financiado pelo Projeto CNPq/Universal 401519/2016-3

${ }^{1}$ Aqui usamos a terminologia de [Bondy and Murty 2008].
} 
menor quantidade de cores em uma b-coloração coincide com o número cromático, e portanto só trabalhamos com o parâmetro de maximização. Em [Irving and Manlove 1999] é definido o número b-cromático de $G$, que é denotado por $b(G)$, como o maior natural $k$ tal que $G$ tem uma b-coloração que usa $k$ cores. No mesmo artigo os autores mostraram que o problema de achar $b(G)$ é NP-completo.

Considerando uma b-coloração com $b(G)$ cores, cada b-vértice claramente tem grau maior ou igual $b(G)-1$. Assim, definindo $m(G)$ como o maior inteiro $k$ tal que $G$ possui $k$ vértices de grau maior ou igual $k-1$, segue que $m(G) \geq b(G)$. Note que pode-se calcular $m(G)$ em tempo polinomial. Irving e Manlove provaram que calcular o número b-cromático de árvores é polinomial. Eles demonstraram primeiro que $b(G) \geq m(G)-1$ e depois que pode-se decidir se $b(G)=m(G)$ em tempo polinomial. Estes resultados foram generalizados em [Campos et al. 2015] para grafos de cintura pelo menos 7. Tais estudos sugerem que grafos de cintura alta são mais fáceis de lidar no que diz respeito à b-coloração.

Irving and Manlove observaram que o cubo tem b-coloração com 2 cores e com 4 cores, mas não possui b-coloração com 3 cores. Inspirado nisso, em [Kratochvíl et al. 2002] é mostrado que, para $n \geq 1$ inteiro, o grafo obtido de $K_{n, n}$ pela deleção de arestas de um emparelhamento perfeito possui b-colorações usando 2 e $n$ cores, mas não com uma quantidade de cores estritamente entre esses dois números. Daí surge a definição do $b$-espectro de $G$, que é o conjunto dos inteiros $k$ tais que $G$ tem uma b-coloração com $k$ cores; tal conjunto é denotado por $S_{b}(G)$. Naturalmente surge também a definição de b-continuidade: dizemos que um grafo é b-contínuo se $S_{b}(G)=[\chi(G), b(G)] \cap \mathbb{Z}$. Em [Barth et al. 2007] é provado que para todo subconjunto finito $S \subset \mathbb{N} \backslash\{1\}$, existe $G$ tal que $S_{b}(G)=S$, e também que o problema de decidir se um dado grafo $G$ é b-contínuo é NP-completo ainda que sejam dadas bcolorações com $\chi(G)$ e $b(G)$ cores. Existem muitos resultados positivos no que diz respeito à b-continuidade de grafos de alguma família. É demonstrado que grafos regulares com cintura maior ou igual a 6, sem ciclos de tamanho 7 são b-contínuos em [Balakarishnan and Kavaskar 2012], e, mais recentemente, que grafos de cintura pelo menos 10 são b-contínuos [Sales and Silva 2017]. Lá, os autores, propõem a seguinte questão: Qual é o menor inteiro $\hat{g}$ tal que $G$ é b-contínuo sempre que tem cintura pelo menos $\hat{g}$. Usando o resultado lá demonstrado, tem-se $\hat{g} \leq 10$. Por outro lado, como $K_{n, n}$ menos um emparelhamento perfeito não é b-contínuo temos que $5 \leq \hat{g}$. Até onde sabemos, nenhum grafo de cintura pelo menos 5 que não é b-continuo é conhecido. O principal resultado desse artigo que melhora o resultado provado em [Sales and Silva 2017] é.

Teorema 1. Se Gé um grafo de cintura pelo menos 8 , então $G$ é b-contínuo.

$\mathrm{E}$, ainda na direção de mostrar que b-colorações de grafos com cintura alta são fáceis, demonstramos o seguinte teorema:

Teorema 2. Se G é um grafo de cintura pelo menos 7 , então $[2 \chi(G), b(G)] \cap \mathbb{Z} \subseteq S_{b}(G)$

De maneira geral, a prova consiste em, dada uma b-coloração com $k$ cores, obter uma b-coloração com $k-1$ cores usando recolorações. Mencionamos que as provas possuem um passo não construtivo, o que é de esperar já que calcular o número cromático de grafos com cintura ao menos $k$ é $N P$-completo, para todo $k \geq 3$ fixo [Lozin and Kaminski 2007].

As seguintes definições serão essenciais para a prova. A cintura de um grafo é o tamanho do seu menor ciclo. Para $z$ inteiro, e $u \in V(G), N_{\leq z}(u)$ denota o conjunto de 
vértices à distância no máximo $z$ de $u$. Em [Sales and Silva 2017], um vértice $u \in V(G)$ é definido para ser uma $k$-íris se existe $S \subseteq N(u)$ tal que $|S| \geq k-1$ e $d(v) \geq k-1$ para todo $v \in S$. Dada $\psi$ uma $k$-coloração de $G$, cada $i \in\{1, \ldots, k\}$ é chamado de cor, enquanto que $\psi^{-1}(i)$ é chamado de classe de cor. Dizemos que um vértice $u$ realiza a cor $i$, se $u \in \psi^{-1}(i)$ e $u$ é b-vértice; dizemos também que $i$ é realizada por $u$. Para $x \in V(G)$ e $i \in\{1, \ldots, k\}, N^{i}(x)=\{v \in N(x) \mid \psi(v)=i\}$, ou seja, os vizinhos de $x$ que tem cor $i$. Para $X \subseteq V(G), N^{i}(X)=\cup_{x \in X} N^{i}(x) \backslash X$. Denotamos por $B(\psi)$ o conjunto de b-vértices de $\psi$ e, para $i \in\{1, \ldots, k\}, B_{i}$ é o conjunto de b-vértices da cor $i$, isto é, $B_{i}=B(\psi) \cap \psi^{-1}(i)$. Para cada $x \in V(G) \backslash B(\psi)$, seja $U(x)=\{i \in$ $\left.\{1, \ldots, k\} \mid N^{\psi(x)}\left(B_{i}\right)=\{x\}\right\}$, ou seja, o conjunto de cores que dependem de $x$ para possuirem b-vértices. Para finalizar, para uma cor $j \in\{1, \ldots, k\}$, dizemos que $x \in V(G)$ é $j$-mutável se existe cor $c$ tal que, ao mudarmos a cor de $x$ para $c$, não criamos b-vértices da cor $j$; caso contrário $x$ é dito j-imutável. Em [Balakarishnan and Kavaskar 2012], é provado o seguinte lema:

Lema 1. Seja $G$ um grafo de cintura pelo menos 6 e sem ciclos de tamanho 7. Se G tem uma $k$-íris com $k \geq \chi(G)$, então $G$ tem uma b-coloração com $k$ cores.

Dado $x \in V(G) \backslash B(\psi)$, quando $U(x)=\emptyset$, podemos mudar a cor de $x$ para uma cor livre sem perder a b-coloração com $k$ cores. Em nossa prova, nos preocupamos especialmente com vértices $x \in V(G) \backslash B(\psi)$ tais que $|U(x)| \geq 2$; a esses chamamos de úteis. Para um conjunto $K \subseteq V(G) \backslash B(\psi)$ monocromático de cor $i$, diremos que uma cor $j$ é dependente de $K$ se $N^{i}\left(B_{j}\right) \subseteq K$. Note que, ao mudarmos as cores de $K$ para quaisquer cores diferente da inicial, perderemos b-vértices de todas as cores dependentes de $K$ e somente destas. Note que se $j$ depende de $K$, então $B_{j} \subseteq N^{j}(K)$.

O teorema principal do artigo e de onde os Teoremas 1 e 2 são corolários é o seguinte.

Teorema 3. Seja $G=(V, E)$ um grafo com cintura maior ou igual a 7. Se G possui uma $b$-coloração com $k$ cores, onde $k \geq \chi(G)+1$, então $G$ possui uma b-coloração com $k-1$ cores oи uта $(k-1)$-íris.

Acreditamos que as técnicas de recoloração alcançam seu limite quando a cintura chega em 7, tanto que o resultado que calcula o número cromático de grafos com cintura 7 apresentado em [Campos et al. 2015] até hoje ainda não foi melhorado. Desta maneira, uma tentativa de encontrar grafos com cintura 6 que não sejam b-contínuos é válida, mesmo se restringimos para grafos bipartidos. Em particular, uma resposta positiva para ambas as perguntas abaixo definiria o valor de $\hat{g}$ em 7 .

Pergunta 1. O Lema 1 pode ser generalizado de maneira a permitir ciclos de tamanho 7 ?

Pergunta 2. Existe grafo bipartido com cintura 6 que não seja b-contínuo?

\section{Idéia da Prova}

Nossa prova segue semelhante à feita em [Sales and Silva 2017], porém damos atenção a uma cor que queremos retirar. Isso faz com que os b-vértices das outras cores se aproximem dos b-vértices dessa cor específica. Seja $\psi$ uma coloração com $k$ cores de $G$. Suponha que $\psi$ minimiza em primeiro lugar a quantidade de b-vértices da cor $1 \mathrm{e}$, em segundo lugar, que minimiza a quantidade de vértices da cor 1 . Seja $u \in B_{1}$. Tentamos recolorir $N(u)$ de forma que $u$ deixe de ser b-vértice, tomando cuidado de não criar mais 
b-vértices da cor 1 e de que todas as outras cores ainda sejam realizadas. Daí, pela minimalidade de $\left|B_{1}\right|$, essa nova coloração não é b-coloração e, como todas as cores que não 1 são realizadas, a cor 1 é a única não realizada. Pela limpeza de 1 , conseguimos uma b-coloração com $k-1$ cores. Argumentamos também que, quando não conseguimos isso, tem-se que $u$ é $k$-íris e, em particular, $(k-1)$-íris; daí a b-coloração desejada é obtida pelo Lema 1. Um fato importante e que já nos direciona para um entendimento do que está acima, é o seguinte.

Fato 1. Para cada cor $j \in\{2, \ldots, k\}$, tem-se que todo $x \in N^{j}(u) \backslash B(\psi)$ é 1-mutável. Além disso, vale um dos seguintes itens:

1. Existe $v \in N(u) \cap B_{j}$;

2. Existe cor $d \in\{2, \ldots, k\} \backslash\{j\}$ tal que $d$ depende de $N^{j}(u)$.

Após a prova do fato, argumentamos que se a quantidade de cores que segue o item 2 é não nula, passando por algumas colorações parciais conseguimos uma b-coloração com $k-1$ cores. Sobrando então o caso onde o item 1 vale para todas as cores. É trivial ver que temos uma $(k-1)$-íris.

\section{Referências}

Balakarishnan, R. and Kavaskar, T. (2012). b-coloring of kneser graphs. Discrete Applied Mathematics, 160:9-14.

Barth, D., Cohen, J., and Faik, T. (2007). On the b-continuity property of graphs. Discrete Applied Mathematics, 155:1761-1768.

Bondy, J. and Murty, U. (2008). Graph Theory. Springer.

Campos, V., Lima, C., and Silva, A. (2015). Graphs of girth at least 7 have high bchromatic number. European Journal of Combinatorics, 48:154-164.

Irving, R. W. and Manlove, D. (1999). The b-chromatic number of a graph. Discrete Applied Mathematics, 91:127-141.

Kratochvíl, J., Tuza, Z., and Voigt, M. (2002). On the b-chromatic number of graphs. In Goos, G., Hartmanis, J., van Leeuwen, J., and Kučera, L., editors, Graph-Theoretic Concepts in Computer Science, pages 310-320. Springer Berlin Heidelberg.

Lozin, V. and Kaminski, M. (2007). Coloring edges and vertices of graphs without short or long cycles. Contributions do Discrete Mathematics, 2.

Sales, C. L. and Silva, A. (2017). The b-continuity of graphs with large girth. Graphs and Combinatorics, 33:1138-1146. 